\title{
Comparative study of iliac crest and proximal femur histomorphometry in normal patients
}

\author{
N L FAZZALARI, R J MOORE, B A MANTHEY, B VERNON-ROBERTS \\ From the Institute of Medical and Veterinary Science, Adelaide, South Australia
}

SUMMARY The relation between the bone histomorphometric profile of the iliac crest and regions of the proximal femur was investigated. In the proximal femur four blocks were selected, representing the subchondral principal compressive (SPC) and tensile (SPT) regions, the zone near the medial cortex in the principal compressive region (MPC), and the zone medial to the greater trochanter (MGT). Undecalcified tissue sections stained by the von Kossa silver method and counterstained with haematoxylin and eosin were used for quantitation. The SPT region was most representative of the iliac bone profile, in particular the structural parameters of mineralised bone, trabecular thickness, and trabecular spacing and resorption surface. Most parameters in the regions studied showed inconsistent and variable results when compared with those of the iliac crest. There was only a weak association, therefore, between the histomorphometric parameters of the iliac crest and the proximal femur despite the appeal of the use of the iliac biopsy specimen as a simple clinical test.

All synovial joints rest on bony foundations, one or both of which primarily comprise cancellous (trabecular) bone. In the case of the proximal femur, the structural and organisational properties of the cancellous tissue could influence the natural lubrication process of the hip joint that occurs at the cartilage interface. Substantial changes occur in this bony tissue in osteoarthritis and could be the initial step in the progression of this disease.'

Osteoporotics have acute subcapital and trochanteric femoral fracture as a consequence of altered trabecular microstructure. ${ }^{2}$ Arthroplasties on diseased joints, which use either cement or bony ingrowth for fixation, also rely on cancellous bone for a substantial portion of their structural support. ${ }^{34}$

Many clinical investigations, including biochemical studies and bone mass measurement, may be used to determine the risk of femoral fracture, but their value is often limited. Radiological assessment for instance, is frequently used, although it is reported that clinically important bone loss may occur before a decrease in bone density is observed radiologically ${ }^{5}$ Furthermore, the radiological Singh index was shown to have no correlation with the bone content of the femoral head, ${ }^{6}$ and for this reason the onset of bone loss may be overlooked. By the time clinical symptoms are detected, the condition may have reached a critical stage.

Accepted for publication 2 March 1989
During osteoarthritis of the hip, substantial changes occur in the tissues which make up its geometry and anatomy, particularly degenerative changes of the subchondral trabecular bone which supports virtually all the load on the joint, and changes in the articular cartilage, the weight bearing surface in the joint. ${ }^{7}$

Histomorphometric analysis of bone from the iliac crest is an acceptable and reliable method of screening patients for their bone state. ${ }^{8}$ Investigators can observe the characteristics of both cortical and trabecular bone, and it also allows dynamic studies of the bone mineralisation process to be done. The method is relatively painless and is highly reproducible. ${ }^{9}$

In this context then, an understanding of the relation between bone histomorphometric profile of the iliac crest and regions of the proximal femur would provide invaluable diagnostic and prognostic information for the treatment of patients. The simple iliac bone biopsy procedure could be used as an accurate screening test for the likelihood of femoral neck fracture, osteoarthritis, or for the evaluation of bone stock quality in the case of arthroplasties performed on the proximal femur, thus enabling therapeutic management.

\section{Material and methods}

The subjects in this study were selected from routine necropsy cases showing no evidence of malignancy or 


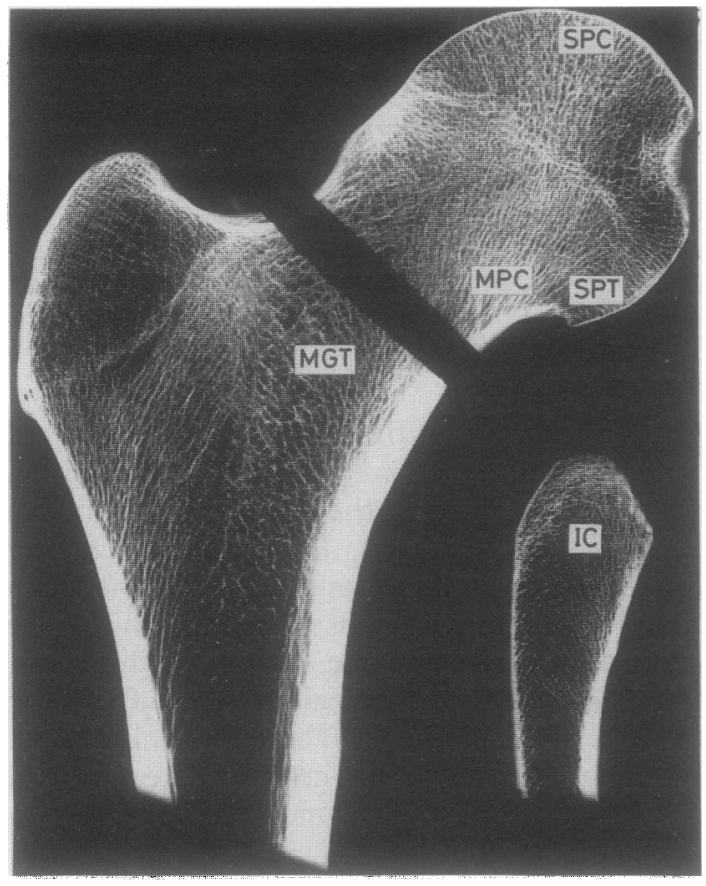

Figure Blocks taken from the proximal femur and the iliac crest wedge for bone histomorphometry. SPC = subchondral bone in the principal compressive region; $M P C=$ bone in the principal compressive region near the medial cortex; $S P T=$ subchondral bone in the principal tensile region; $M G T=$ bone medial to the greater trochanter; IC = iliac crest.

any other abnormality. The age of the subjects ranged from 18 to 90 years. There were 14 men, aged 44.1 (SD $24 \cdot 1)$ years, and eight women, aged $43.0(19 \cdot 3)$ years. The proximal femur was excised from each subject at the level of the lesser trochanter and placed in $10 \%$ neutral buffered formalin. A wedge of bone was also taken from the iliac crest at a point $3 \mathrm{~cm}$ posterior to the anterior superior iliac spine and similarly fixed. All samples were taken from the right side of the skeleton.

At a later stage, a $5 \mathrm{~mm}$ thick coronal slice was taken through the fovea of the femoral head using a bandsaw. Four smaller blocks were then selected from this slice, representing the subchondral principal com- pressive (SPC) and subchondral principal tensile (SPT) regions, the zone near the medial cortex in the principal compressive region (MPC), and the zone medial to the greater trochanter (MGT) (figure).

All blocks were processed undecalcified into Araldite epoxy resin (Ciba Geigy, Melbourne, Australia) and sectioned on a Jüng $K$ microtome (Reichert Jüng, Heidelberg, West Germany). Three, $8 \mu$ m thick sections, spaced at about $200 \mu \mathrm{m}$ intervals, were stained by the von Kossa silver method and counterstained with haematoxylin and eosin to distinguish between the mineralised bone, the osteoid, and the cellular components of the marrow. Histoquantitation was performed by one technician using an ocular-mounted Weibel II graticule, at a magnification of 100 times.

Estimates were made of the following parameters: percentage of mineralised bone (BV/TV); surface density of bone in $\mathrm{mm}^{2} / \mathrm{mm}^{3}$ (BS/TV); specific surface of bone in $\mathrm{mm}^{2} / \mathrm{mm}^{3}(\mathrm{BS} / \mathrm{BV})$; trabecular thickness in $\mathrm{mm}$ (Tb.Th); trabecular spacing in $\mathrm{mm}$ (Tb.Sp); percentage of osteoid volume (OV/TV); percentage of osteoid surface (OS/BS); percentage of resorbing surface $(\mathrm{ES} / \mathrm{BS})$. These indices are described in full in a recent publication. ${ }^{10}$

A one way analysis of variance was performed on the data to determine whether differences existed between the iliac crest bone and selected regions of the proximal femur. The Neuman-Keuls test was then used to test for significant differences between the iliac crest and the femoral head regions. Significance was set at $p<0.05 .1$

\section{Results}

The histomorphometric estimates from each sample are listed separately for men (table 1) and women (table 2), and for the combined population (table 3). The significance of the differences between the iliac crest sample and the four regions of the femoral head is indicated in each table.

In the male subgroup estimates of BV/TV from the iliac crest did not differ significantly from the femoral head regions except for the MPC region. Estimates of both OV/TV and ES/BS in the iliac crest did not differ significantly from any of the femoral head regions,

Table 1 Bone histomorphometry of regions in proximal femur and iliac crest in men (figures in parentheses indicate SD)

\begin{tabular}{|c|c|c|c|c|c|}
\hline & $S P C$ & $M P C$ & $S P T$ & $M G T$ & $I C$ \\
\hline $\begin{array}{l}\text { BV/TV } \\
\text { BS/TV } \\
\text { BS/BV } \\
\text { Tb.Th } \\
\text { Tb.Sp } \\
\text { OV/TV } \\
\text { OS/BS } \\
\text { ES/BS }\end{array}$ & $\begin{array}{l}26.9(8.3) \\
2.54(0.37) \\
10.5(4 \cdot 2)^{*} \\
0.423(0 \cdot 114)^{*} \\
1.185(0.260) \\
0.156(0.143) \\
8.4 \quad(6.8)^{*} \\
1.7 \quad(1.1)\end{array}$ & $\begin{array}{l}32.9(7.4)^{*} \\
2.19(0.68)^{*} \\
6.8(2 \cdot 0)^{*} \\
0.656(0.228)^{*} \\
1.337(0.415) \\
0.108(0.103) \\
5.7 \quad(3.4)^{*} \\
2.4 \quad(1.4)\end{array}$ & $\begin{array}{c}21.2(8.1) \\
2.58(0.61) \\
13.5(4.4) \\
0.325(0.100) \\
1.317(0.472) \\
0.448(0.930) \\
10.6 \quad(9.0)^{*} \\
2.9 \quad(1.7)\end{array}$ & $\begin{array}{l}16.6(9.5) \\
1.82(0.47)^{*} \\
13.6(5.4) \\
0.352(0.174) \\
2.023(0.843)^{*} \\
0.104(0.166) \\
9.5 \quad(9.9)^{*} \\
2.4 \quad(1.7)\end{array}$ & $\begin{array}{c}19.4(7.6) \\
2.87(0.62) \\
16.7(5.8) \\
0.263(0.081) \\
1.200(0.401) \\
0.502(0.338) \\
18.8 \quad(10.2) \\
3.2 \quad(1.7)\end{array}$ \\
\hline
\end{tabular}

$* \mathrm{p}<0.05$. 
Table 2 Bone histomorphometry of regions in proximal femur and iliac crest in women (figures in parentheses indicate SD)

\begin{tabular}{|c|c|c|c|c|c|}
\hline & $S P C$ & $M P C$ & $S P T$ & $M G T$ & $I C$ \\
\hline $\begin{array}{l}\text { BV/TV } \\
\text { BS/TV } \\
\text { BS/BV } \\
\text { Tb.Th } \\
\text { Tb.Sp } \\
\text { OV/TV } \\
\text { OS/BS } \\
\text { ES/BS }\end{array}$ & $\begin{array}{l}30.3(2.5)^{*} \\
2.72(0.50)^{*} \\
9.0(1.4)^{*} \\
0.458(0.087)^{*} \\
1.059(0.213) \\
0.144(0.097)^{*} \\
10.3 \quad(5.5) \\
2.9 \quad(2.5)\end{array}$ & $\begin{array}{l}32.3(5.1)^{*} \\
2.15(0.53)^{*} \\
6.7(1.3)^{*} \\
0.620(0.125)^{*} \\
1.348(0.446) \\
0.060(0.047)^{*} \\
6.1 \quad(3.2) \\
3.6 \quad(1.8)\end{array}$ & $\begin{array}{c}17 \cdot 8(5.7) \\
2.78(0.63)^{*} \\
16 \cdot 3(3 \cdot 1)^{*} \\
0.253(0.047) \\
1.257(0.372) \\
0.139(0.136)^{*} \\
10.1 \quad(6.7) \\
2.6 \quad(2.0)\end{array}$ & $\begin{array}{l}12.5(4.6)^{*} \\
1.93(0.62)^{*} \\
16.2(3.5)^{*} \\
0.273(0.074) \\
2.062(0.934)^{*} \\
0.027(0.035)^{*} \\
6.1 \quad(4.2) \\
2.0 \quad(1.5)\end{array}$ & $\begin{array}{c}19.8(4.7) \\
3.78(0.67) \\
19.4(2 \cdot 4) \\
0.210(0.031) \\
0.882(0.201) \\
0.298(0.198) \\
11.6 \quad(7.6) \\
4.0 \quad(1.2)\end{array}$ \\
\hline
\end{tabular}

${ }^{*} \mathrm{p}<0.05$.

whereas OS/BS in all regions of the proximal femur differed significantly from the iliac bone. Of all the regions in the femur, the SPT region was the best estimator of the iliac bone profile.

In the female subgroup the SPT region of the femur was the only area which did not differ significantly from the iliac crest in the estimation of BV/TV. Estimates of BS/TV, BS/BV, and OV/TV in the femoral regions all differed significantly from the iliac crest. On the other hand, estimates of OS/BS and ES/BS showed no significant difference between the iliac and proximal femoral sites. As for the male group the SPT region was the best estimator of the iliac sample for the parameters studied.

When the male and the female data were combined, the differences between the two skeletal regions became more diverse, although estimates of ES/BS at all femoral sites remained insignificantly different from the iliac sample. Estimates of BS/TV, BS/BV, and OS/BS were all significantly different between the femoral regions and the iliac crest. As expected from the sex-grouped data, the SPT region in the femoral head was again the best estimator of iliac histomorphometry for the parameters studied.

In each of the male, female, and combined groups, the bone from the MGT region of the femoral head was the only area which differed significantly from the iliac crest in the estimation of trabecular space. Furthermore, estimates of trabecular thickness from the SPC and MPC regions differed significantly from the iliac crest in all three groups.

\section{Discussion}

Our iliac crest data correspond well with the extensive studies of another group, ${ }^{12}$ although our estimates of the extent of resorption occurring on the trabecular surface are about half of their values. It is difficult to understand why this should be, given that the criteria for evaluating this feature were identical-that is, the percentage of trabecular surface displaying Howship's lacunae (with or without osteoclasts). This may simply be an extreme case of interobserver variation.

The principal purpose of this investigation, however, was to establish whether the bone of the iliac crest is representative of that in the proximal femur. The four regions selected from the femoral head represented the sites affected in both degenerative joint disease and proximal femoral fracture. They are diverse in their mechanical and physical characteristics, and as such may be expected to show a diversity of histological and histomorphometric features.

Perhaps the most interesting finding in this study was the lack of significant difference between estimates of ES/BS from all femoral head regions and the iliac crest. This result indicates that the resorption phenomenon is occurring at a constant level at all of these sites. This is especally interesting in view of the fact that these regions are under different mechanical stresses and therefore have adopted different architectural features, as shown by the other structural parameters studied. The bone of the iliac crest, for example, takes the load of the whole upper body

Table 3 Bone histomorphometry of regions in proximal femur and iliac crest in men and women (figures in parentheses indicate $S D$ )

\begin{tabular}{|c|c|c|c|c|c|}
\hline & $S P C$ & $M P C$ & $S P T$ & $M G T$ & $I C$ \\
\hline $\begin{array}{l}\text { BV/TV } \\
\text { BS/TV } \\
\text { BS/BV } \\
\text { Tb.Th } \\
\text { Tb.Sp } \\
\text { OV/TV } \\
\text { OS/BS } \\
\text { ES/BS }\end{array}$ & $\begin{array}{l}28 \cdot 1(6.9)^{*} \\
2.60(0.42)^{*} \\
9.9(3.5)^{*} \\
0.436(0.104)^{*} \\
1.139(0.246) \\
0.152(0.126)^{*} \\
9.1 \quad(6.3)^{*} \\
2.2 \quad(1.8)\end{array}$ & $\begin{array}{l}32.7(6.5)^{*} \\
2.17(0.62)^{*} \\
6.8(1.7)^{*} \\
0.643(0.194)^{*} \\
1.341(0.416) \\
0.090(0.089)^{*} \\
5.8 \quad(3.2)^{*} \\
2.8 \quad(1.6)\end{array}$ & $\begin{array}{l}20.0(7.4) \\
2.65(0.61)^{*} \\
14.5(4.1)^{*} \\
0.299(0.091) \\
1.295(0.430) \\
0.335(0.751) \\
10.4 \quad(8.1)^{*} \\
2.8 \quad(1.8)\end{array}$ & $\begin{array}{l}15.1(8.2) \\
1.86(0.52)^{*} \\
14.5(4.9)^{*} \\
0.323(0.149) \\
2.037(0.855)^{*} \\
0.076(0.137)^{*} \\
8.3 \quad(8.3)^{*} \\
2.3 \quad(1.6)\end{array}$ & $\begin{array}{c}19.5(6.6) \\
3.20(0.76) \\
17.7(4.9) \\
0.244(0.071) \\
1.084(0.371) \\
0.428(0.307) \\
16.2 \quad(9.8) \\
3.5 \quad(1.6)\end{array}$ \\
\hline
\end{tabular}

$* 0.05$. 
across a broad flat area, whereas the proximal femur bears the transferred load on a smaller and much more specialised structure.

The study also indicates an apparent sex-linked difference between osteoid surface and volume parameters, consistent across all regions of the femoral head. Estimates of OV/TV in men did not differ significantly between iliac crest and femoral head regions, while in the female population the converse was true. The estimates of OS/BS in men were significantly different across these regions, but not in the case of women.

The remaining parameters show inconsistent and variable results with no true pattern to the relations between any of the sites. If any one region could be described as correlating reasonably well with the iliac crest, however, it must be the SPT region. Even though this area is not directly associated with fractures of the femoral neck and total hip replacement, studies of this area show that measurement of bone structural parameters and bone turnover indices are useful indicators of the state of the femoral head. During severe osteoarthritis, this region undergoes extensive bony exchanges and further studies are required to determine whether these changes are purely local or linked to systemic bony changes. ${ }^{1314}$

This histomorphometric study of cancellous bone from the iliac crest and proximal femur highlights the fundamental importance of the need for a greater understanding of trabecular bone structure. It is not sufficient simply to measure the mineralised bone volume and extrapolate from this the nature of bone structure or its mechanical properties. Table 1, for example, shows that the iliac crest mineralised bone volume is representative of most regions, yet the bone is spatially disposed in a number of geometries. On the other hand, the bone turnover indices tend to be more uniform, showing no statistical difference for ES/BS in all groups and no difference for OS/BS for women and OV/TV for men.

Overall, there seems to be only a weak association between the histomorphometric parameters of the iliac crest and the proximal femur, and despite the appeal of the iliac biopsy specimen as a simple clinical test, its diagnostic value as a predictor of femoral neck fracture, osteoarthritis, and for the evaluation of bone stock quality in the case of proximal femoral arthroplasties is poor.

Supported by a grant from the Adelaide Bone and Joint Research Foundation and the National Health and Medical Research Council.

\section{References}

1 Pugh JW, Radin EL, Rose RM. Quantitative studies of human subchondral cancellous bone: its relationship to the state of its overlying cartilage. J Bone Joint Surg 1974;56A:313-21.

2 Melton LJ, Chao EYS, Lane J. Biomechanical aspects of fractures. In: Riggs BL, Melton LJ, eds. Osteoporosis-etiology, diagnosis and management. New York: Raven Press Ltd, 1988:111-32.

3 Hvid I. Mechanical strength of trabecular bone at the knee. Danish Medical Bulletin 1988;35:345-65.

4 Saito M, Saito S, Ohzono K, Ono K. The osteoblastic response to osteoarthritis of the hip. J Bone Joint Surg 1987;69B:746-51.

5 McCarthy P. Hip fractures in elderly women. Med J Aust 1985;143:372.

6 Wicks M, Garrett R, Vernon-Roberts B, Fazzalari N. Absence of metabolic bone disease in the proximal femur in patients with fracture of the femoral neck. J Bone Joint Surg 1982;64B: 319-22.

7 Byers PD, Contepomi CA, Farkas TA. A postmortem study of the hip joint: including the prevalence of the features of the right side. Ann Rheum Dis 1970;29:15-31.

8 Parfitt AM. The physiologic and clinical significance of bone histomorphometric data. In: Recker RR, ed. Bone histomorphometry: techniques and interpretation. Boca Raton: CRC Press, 1983:143-224.

9 Rao DS. Practical approach to bone biopsy. In: Recker RR, ed. Bone histomorphometry: techniques and interpretation. Boca Raton: CRC Press, 1983:3-12.

10 Parfitt AM, Drezner MK, Glorieux FH, et al Bone histomorphometry: Standardization of nomenclature, symbols and units. Report of the ASMBR Histomorphometry Nomenclature Committee. J Bone Mineral Res 1987;2:595-610.

11 Snedecor GW, Cochran WG. Statistical methods. Ames, Iowa: Iowa State University Press, 1980.

12 Merz WA, Schenk RK. Quantitative structural analysis of human cancellous bone. Acta Anat 1970;75:54-66.

13 Fazzalari NL, Vernon-Roberts B, Darracott J. Osteoarthritis of the hip: possible protective and causative roles of trabecular microfractures in the head of the femur. Clin Orthoped 1987;216:224-33.

14 Solomon L, Schnitzler CM, Browett JP. Osteoarthritis of the hip: the patient behind the disease. Ann Rheum Dis 1982;41:118-25.

Requests for reprints to: Dr N L Fazzalari, Institute of Medical and Veterinary Science, Box 14 Rundle Mall PO, Adelaide SA 5000, Australia. 